This is an author produced version of a paper published in Applied Radiation and Isotopes. This paper has been peer-reviewed but does not include the final publisher proof-corrections or journal pagination.

Citation for the published paper:

Hjerpe, Thomas and Samuelsson, Christer

"Shielded and unshielded geometries in the search for orphan sources"

Appl Radiat Isot. 2006 May;64(5):551-5.

http://dx.doi.org/10.1016/j.apradiso.2005.11.017.

Access to the published version may require journal subscription.

Published with permission from: Elsevier 


\title{
Shielded and unshielded geometries in the search for Orphan Sources
}

\author{
Thomas Hjerpe and Christer Samuelsson \\ Department of Medical Radiation Physics, The Jubileum Institute, \\ Lund University, University Hospital, SE-221 85 Lund, Sweden
}

\begin{abstract}
A car-borne $\mathrm{NaI}(\mathrm{Tl})$ spectrometric system was used together with a ${ }^{137} \mathrm{Cs}$ source to obtain realistic data in the search of unshielded and semi-shielded orphan sources. The PSC (potassium stripped counts) method was used to estimate the influence by the shielding on the detection ability. A reduction of about $5 \%$ in the critical distance was obtained for the semi-shielded source. A curve fitting method was also developed and evaluated. The results showed inferior ability to find the source, compared to the PSC method. However, it can be a useful complementary tool, for characterization of the source shielding, and estimation of the distance from the road.
\end{abstract}

Keywords: Orphan sources; Radioactive source search; Mobile gamma spectrometry

\section{INTRODUCTION}

The definition of an orphan source is according to the International Atomic Energy Agency (IAEA), (2003): "A source which poses sufficient radiological hazard to warrant regulatory control, but is not under regulatory control because it has never been so, or because it has been abandoned, lost, misplaced, stolen or otherwise transferred without proper authorization". In the European Union (EC, 2003), an orphan source is further specified as a sealed source, with an activity level of which, at the time of its discovery, is above the exemption level. An important component in the emergency preparedness strategy is the use of monitoring systems to detect orphan sources; of which mobile systems are a subtype. The radiation detector can be mounted in a car (as in this study) or other vehicles, such as fixed-wing aircrafts or helicopters. With mobile systems, a large area can be scanned in a short time. Any detector sensitive to gamma radiation can be used, but highly sensitive gamma spectrometers are to prefer. Successful use of mobile gamma spectrometers in the search for orphan radioactive sources, and the recovery of radioactive material in the environment have been demonstrated over the years (e.g., Deal et al., 1972; Bristow, 1978; Moreira, 1991).

The main challenge when searching for orphan sources with mobile systems is to distinguish between signals originating from an actual source and signals originating 
from the natural background radiation. The task is complicated by the fact that the measurements take place in an environment with varying background radiation levels. Any criterion for deciding that a measured radiation field indicates the presence of an orphan source has to consider this variable background. The strategy used by the search team is dependant on the information available on the source. Important information is knowledge about the radionuclide composition and strength, and indications of the source location. For example, if the scenario is a lost therapy source from a hospital, good knowledge about the characteristics of the source is most likely available. However, the source can be very well shielded and difficult to detect, or partly shielded. This study is focused on a scenario with known source characteristics, and a geometry where the source in either unshielded or semi-shielded. The semishielded geometry represents a scenario where, e.g., the source is located next to a house or behind a rock. The source used was a ${ }^{137} \mathrm{Cs}$ source with an activity of $2 \mathrm{GBq}$. In a scenario with a single gamma emitting radionuclide and known source characteristics, the search can be focused on one or a few gamma energies. This justifies the use of a low-resolution detector system. Here, a car-borne gamma spectrometry system equipped with a $4129 \mathrm{~cm}^{3} \mathrm{NaI}(\mathrm{Tl})$ detector was utilized.

An on-line strategy that has been proven useful in a scenario with an unshielded orphan source is the PSC method (Hjerpe and Samuelsson, 2003). The PSC method is a robust and reliable method, based on a moving average algorithm that responds to significant increases in the amplitude of the ${ }^{137} \mathrm{Cs}$ full energy peak signal. A potential weak point with the PSC method is that it does not utilize that the expected curve shape, of the signal originating from the point source, in consecutive measurements when passing an unshielded point source can be theoretically determined. In addition, the results from the PSC method do not give an estimation of the source to road distance. A curve fitting method was applied on the data to test if the ability to detect the source can be increased by assuming a curve shape of the measured signal, where the source strength is given. A second application for curve fitting was also tested: use it as complementary tool to the PSC method, for characterization of the source shielding geometry on found sources without the need for leaving the vehicle. When the source was regarded to be unshielded, curve fitting was also used to estimate the source to road distance.

The objectives are to evaluate if the ability of finding an orphan source is affected by a semi-shielded geometry, and investigate if a method based on curve fitting can be an alternative search method. Furthermore, evaluate if curve fitting can be used to give information about the shielding geometry and for estimation of the source to road distance of an orphan source found by the PSC method.

\section{THEORY AND METHODS}

The parameters used from the spectra are the measured counts in the energy windows for the $661.6 \mathrm{keV}$ gamma line of ${ }^{137} \mathrm{Cs}$, and the $1461 \mathrm{keV}$ gamma line of ${ }^{40} \mathrm{~K}$. The counts in the ${ }^{137} \mathrm{Cs}$ originating from ${ }^{40} \mathrm{~K}$ is subtracted, using the stripping ratio $c_{\mathrm{K}, \mathrm{Cs}}$ 
(the ratio between the number of counts caused by ${ }^{40} \mathrm{~K}$ in the ${ }^{137} \mathrm{Cs}$ window and the number of counts in the ${ }^{40} \mathrm{~K}$ own window). This approach of deconvoluting the spectral interference from ${ }^{40} \mathrm{~K}$ in the ${ }^{137} \mathrm{Cs}$ window is recommended by the IAEA and the International Commission on Radiation Units and Measurements (ICRU), and is commonly referred to as the Stripping method (IAEA, 1991; ICRU, 1994). To avoid introducing large uncertainties by the short sampling time, the spectral interferences from $\mathrm{U}$ and $\mathrm{Th}$ in the ${ }^{137} \mathrm{Cs}$ window are neglected. When searching for orphan radioactive sources in the environment from a moving vehicle it is important to use a well-thought-out strategy in order to make optimal use of the information obtained. Two different methods to analyse the obtained data are used in this study.

\section{The PSC method}

A straightforward on-line method that has been proven powerful is the PSC method (Hjerpe and Samuelsson, 2003). It is based on a moving average algorithm applied to the 25 most recently consecutively collected spectra, where the sampling time for each spectrum is three seconds. The spectra are divided into a test group containing the five most recently collected spectra and a control group containing the other 20 . The test statistic, $P S C$, is the ratio of the average number of potassium-stripped counts for the test group, $N_{\mathrm{C}, 1 . . .5}^{\prime}$, and the control group, $N_{\mathrm{Cs}, 6 \ldots 25}^{\prime}$. This can be expressed as:

$$
\begin{aligned}
& P S C=\frac{N_{\mathrm{Cs}, 1 \ldots 5}^{\prime}}{N_{\mathrm{Cs}, 6 \ldots 25}^{\prime}} \\
& N_{\mathrm{Cs}}^{\prime}=N_{\mathrm{Cs}}-c_{\mathrm{K}, \mathrm{Cs}} N_{\mathrm{K}}
\end{aligned}
$$

where $N_{\mathrm{Cs}}$ and $N_{\mathrm{K}}$ are the measured gross number of counts in the ${ }^{137} \mathrm{Cs}$ window and ${ }^{40} \mathrm{~K}$ window respectively, and $c_{\mathrm{K}, \mathrm{Cs}}$ is the stripping ratio for the interference due to ${ }^{40} \mathrm{~K}$ in the ${ }^{137}$ Cs window.

The resulting $P S C$ is compared to a pre-estimated alarm level. The alarm level for the PSC method, $L_{\mathrm{PSC}}$, was determined from about 3,000 spectra obtained by car-borne measurements on natural background, using the procedure described by Hjerpe et al. (2001).

\section{Curve fitting}

Driving with a constant speed on a straight road passing a point source and measure continuously with a fixed sampling time is mathematically equivalent to performing stationary measurements of a line source (Coll, 1985). The length and source strength of the equivalent line source is determined by the sampling time of the spectrometer system, the driving speed and the activity of the point source. The equivalent principle allows using the well-known expression for calculating the photon fluence at any 
point in space from a line source (see Fig. 1 for geometric definitions). Thus, the photon fluence, $\Phi$, at the detector during one measurement can be expressed as:

$$
\Phi=\frac{S}{4 \pi D} \int_{\operatorname{atan}\left(\frac{\mathrm{x}}{D}-\frac{\mathrm{L}}{2 D}\right)}^{\operatorname{atan}\left(\frac{\mathrm{x}}{D}+\frac{\mathrm{L}}{2 D}\right)} e^{-\mu D_{\sec }(\theta)} d \theta
$$

where

$S$ is the source strength of the equivalent line source

$D$ is the perpendicular distance from the road to the source

$\mathrm{x}$ is the centre position on the road that is covered by the measurement

$\mathrm{L}$ is the distance covered by one measurement

$\mu$ is the linear attenuation coefficient in air at an energy of $661.6 \mathrm{keV}$

If the response, $\varepsilon(\theta)$, of the detector systems to a ${ }^{137} \mathrm{Cs}$ point source at distance $D$ is known, the net counts in the ${ }^{137} \mathrm{Cs}$ window for a measurement with sampling time $t$ is determined by:

$$
N=t \varepsilon(\theta) \Phi
$$

The measured counts are proportional to the photon fluence, assuming a negligible angular dependency of the response. Eq. 4 can be used to determine the theoretical curve shape of the expected net counts in the ${ }^{137} \mathrm{Cs}$ window, caused by the point source, as function of the position of the road. Using Eq. 4 as regression model is not straightforward. In this study, the regression model was chosen to the first-order serial expansion of Eq. 4. A constant, C, was also added to represent the signal in the ${ }^{137} \mathrm{Cs}$
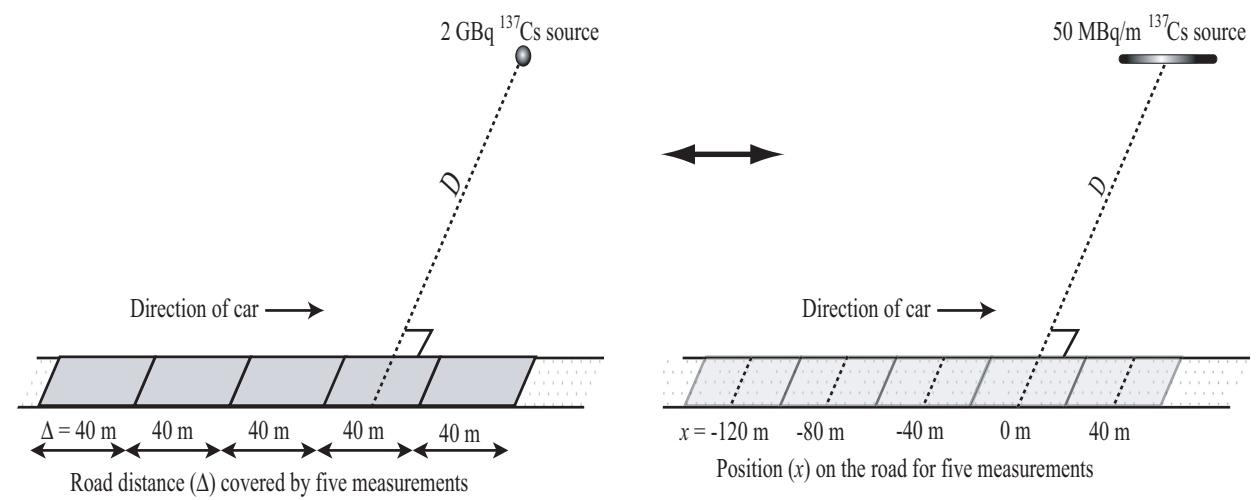

Figure 1. The experimental geometry, and its equivalent situation according to the "duality principle" (Coll, 1985). 
window not originating from the point source. The four-parameter regression model can be written:

$$
N(x)=\frac{t \varepsilon(D) \mathrm{S}}{4 \pi D} \mathrm{e}^{-\mu D}\left(\theta_{1}(\mathrm{x})-\theta_{2}(\mathrm{x})\right)+\mathrm{C}
$$

where

$$
\theta_{1}(\mathrm{x})=\operatorname{atan}\left\{\frac{(\mathrm{x}-\mathrm{B})+\mathrm{L} / 2}{D}\right\} \quad, \quad \theta_{2}(\mathrm{x})=\operatorname{atan}\left\{\frac{(\mathrm{x}-\mathrm{B})-\mathrm{L} / 2}{D}\right\}
$$

The parameters have clear physical interpretations, and are summarized in Table 1.

\section{The curve fitting method}

The regression model above (Eqs. 5 and 6) was here applied as a search method, where the source strength was known. The fitting procedure was applied on estimated $N_{C s}^{\prime}$ in the nine latest consecutive spectra. The test statistic was chosen to the correlation coefficient between the fitted curve and the experimental data points, $R_{\mathrm{yy}(\mathrm{x})}^{2}$. The resulting $R_{\mathrm{yy}(\mathrm{x})}^{2}$ was compared to a pre-estimated alarm level. The alarm level for the curve fitting method ( $C F$ method), $L_{C F}$, was determined from same spectra obtained by car-borne measurements on natural background as for the PSC method, using the procedure described by Hjerpe et al. (2001). However, due to the timeconsuming curve fitting procedure, fewer spectra were used than for estimating $L_{\mathrm{PSC}}$.

\section{Strategy in the search for and characterisation of orphan sources}

The first objective was comparing the PSC and CF methods, when the methods are individually used as primary search algorithms. The critical distance $(C D)$ for the unshielded and the semi-shielded ${ }^{137} \mathrm{Cs}$ source are estimated. The $C D$ is defined as the $D$ at which the source can be detected with $50 \%$ probability when passing it.

The second objective was to use the PSC method as the primary search method. When the PSC method alarm, the curve fitting technique is applied to the resulting $N_{\mathrm{Cs}}^{\prime}$ in the spectra from the interesting area. The resulting $R_{\mathrm{yy}(\mathrm{x})}^{2}$ is used to decide if the source can be regarded as shielded or unshielded. When the source is regarded to

Table 1. Physical interpretations of the regression parameters in Eqs. 5 and 6.

\begin{tabular}{cl}
\hline $\begin{array}{c}\text { Regression } \\
\text { parameter }\end{array}$ & Physical interpretation \\
\hline$S$ & The strength of the equivalent line source $\left[\mathrm{s}^{-1} \mathrm{~m}^{-1}\right]$ \\
$B$ & Position on road with maximum fluence $[\mathrm{m}]$ \\
$C$ & Baseline counts in the ${ }^{137} \mathrm{Cs}$ window, not originating from the point source \\
$D$ & The perpendicular distance from road the to the source $[\mathrm{m}]$ \\
\hline
\end{tabular}


be unshielded, the fitted distance $D$ is then the estimation of the distance between the source and the road.

\section{MATERIALS AND EXPERIMENT}

The gamma spectrometry system used was a GDM 40 RPS system, manufactured by GammaData Burklint $\mathrm{AB}^{(1)}$, in conjunction with a 4-litre $\mathrm{NaI}(\mathrm{Tl})$ detector. The system was energy calibrated, were the window settings was from 545 to $755 \mathrm{keV}$ and from 1320 to $1600 \mathrm{keV}$ for the ${ }^{137} \mathrm{Cs}$ and ${ }^{40} \mathrm{~K}$ windows, respectively.

In the field experiments, a sealed ${ }^{137} \mathrm{C}$ s source with an activity of $2 \mathrm{GBq}$ was acting as the orphan source. Measurements with the source were performed in a flat field on a military training ground near Revingehed, $15 \mathrm{~km}$ east of Lund, Sweden. The system was placed inside the car with the detector mounted approximately $2 \mathrm{~m}$ above the ground to minimize the influence of the roughness of the ground surface. The source was placed unshielded on the field, at $D=100,120,140,160,175$ and $210 \mathrm{~m}$. For each $D$, the source was passed ten times with a driving speed of about $50 \mathrm{~km} / \mathrm{h}$, and measuring with a sampling time of three seconds. The geometry, both the physical situation and the equivalent situation used in the calculations, is described in Figure 1. About 3,000 spectra, without the use of a radioactive source, were also obtained driving in the area; these measurements were used to estimate the alarm levels.

The geometry with a semi-shielded source was produced by manually cropping the data set (see Fig. 2 for an illustrative example). The curve fitting was performed with the software LAB Fit.

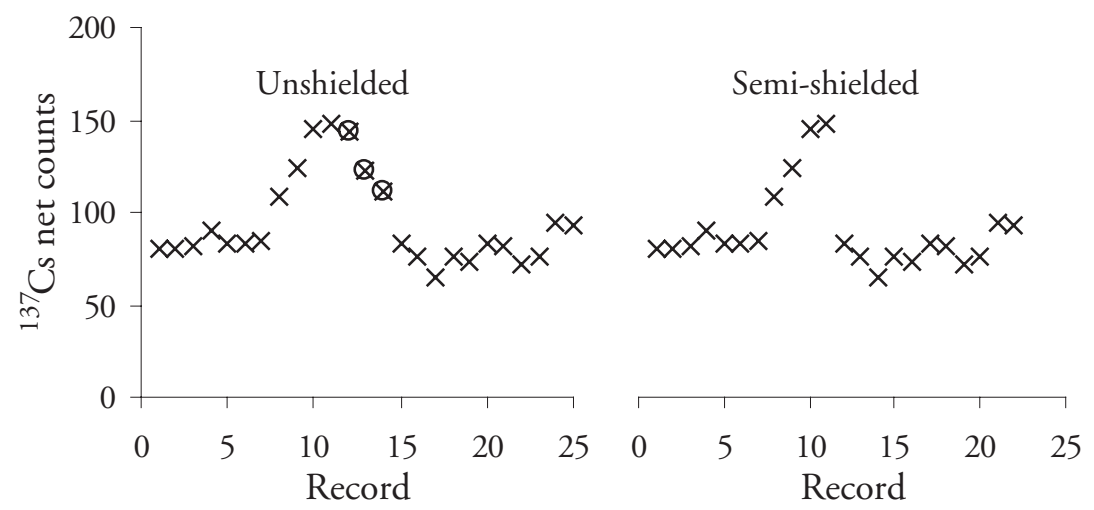

Figure 2. An example of results when passing the unshielded source, and the constructed semi-shielded source. The three data points with circles around are cropped to construct the data set representing a semi-shielded source.

(1) GammaData Burklint AB, Box 15120, Uppsala, Sweden 


\section{RESULTS AND DISCUSSION}

The stripping ratio $c_{\mathrm{K}, \mathrm{Cs}}$ was determined to 0.76 . The alarm levels, $L_{\mathrm{PSC}}$, and $L_{\mathrm{CF}}$ at the one false alarm per hour rate, was determined to 1.38 and 0.86. In the estimation of $L_{\mathrm{CF}}$, requirements were set on the fitted parameters, by the means of allowed ranges (see Table 2). The resulting $R_{y y(x)}^{2}$ were the fitted parameters did not fulfil all requirements were excluded.

\section{Critical distances}

The estimated $C D$ s for the PSC method and the $C F$ method are summarized in Table 3. As seen from the results in Table 3, the PSC method is both more sensitive (higher $C D s$ ) and more robust to shielded geometries, compared to the $C F$ method. The lower $C D$ s for the $C F$ method are not surprising. The few data points, the small increase above to the baseline, the large background variations, and the high uncertainties in the data is not the ideal situation for performing regression analysis. A technique based on smoothing the data, such as the PSC method, is a more logical approach.

\section{Curve fitting as a characterization tool}

In the second objective, using the curve fitting as complementary tool to the PSC method, the curve fitting was only performed when the PSC method gave alarm. The resulting $R_{y y(\mathrm{x})}^{2}$ for both shielding geometries, are plotted against the fitted $D$ in Figure 3. The results in Figure 3 show that unshielded sources up to a $D$ of about 100 to $120 \mathrm{~m}$ can be distinguished from semi-shielded sources, by examine $R_{y y(x)}^{2}$.

For the measurements with the unshielded source, the average fitted source to road distance $\left(D_{\text {fit }}\right)$ is plotted against the actual distance $\left(D_{\text {true }}\right)$ in Figure 4. The result shows fairly good agreement between the fitted distance and actual distance, with better accuracy for the shorter distances. The regression model used underestimates the distance, and the effect is clearer for larger distances. This underestimation is

Table 2. The allowed ranges for the regression parameters.

\begin{tabular}{cc}
\hline $\begin{array}{c}\text { Regression } \\
\text { parameter }\end{array}$ & Range \\
\hline$B$ & -200 to $200 \mathrm{~m}$ \\
$C$ & -25 to 125 counts \\
$D$ & $<250 \mathrm{~m}$ \\
\hline
\end{tabular}

Table 3. Estimated $C D$ s for the $P S C$ and $C F$ methods, for a $2 \mathrm{GBq}{ }^{137} \mathrm{Cs}$ source. Two shielding geometries are considered: unshielded and semi-shielded.

\begin{tabular}{ccccc}
\hline & \multicolumn{2}{c}{ PSC method } & \multicolumn{2}{c}{ CF method } \\
\hline & Unshielded & Semi-shielded & Unshielded & Semi-shielded \\
Critical distance & $180 \mathrm{~m}$ & $170 \mathrm{~m}$ & $150 \mathrm{~m}$ & $<100 \mathrm{~m}^{\mathrm{a}}$ \\
\hline
\end{tabular}

${ }^{a}$ Not detected at the minimum $D$ of $100 \mathrm{~m}$ 


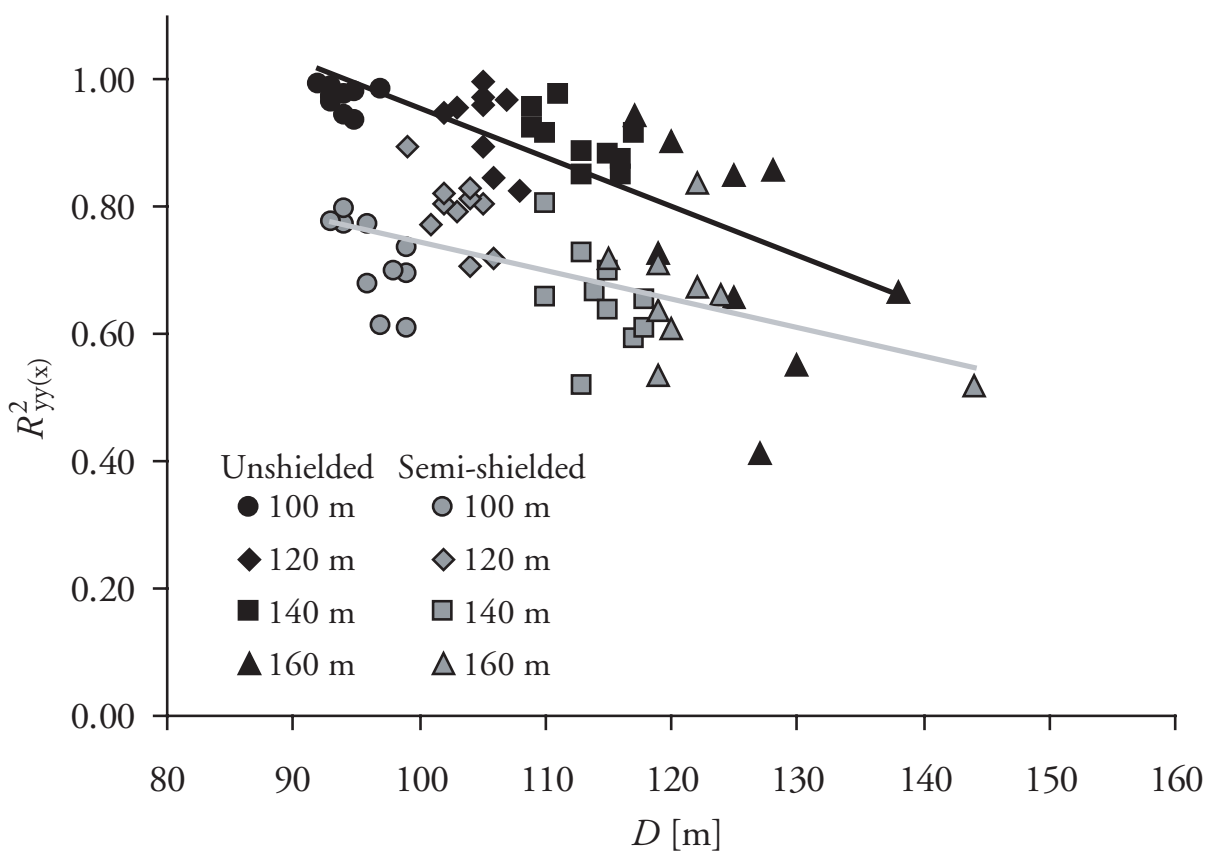

Figure 3. Resulting $R_{y y(x)}^{2}$ as function of fitted $D$ for the true source to road distances 100,120,140, and $160 \mathrm{~m}$; the black markers represent the unshielded geometry and the grey markers the semi-shielded. The lines are linear regression lines.

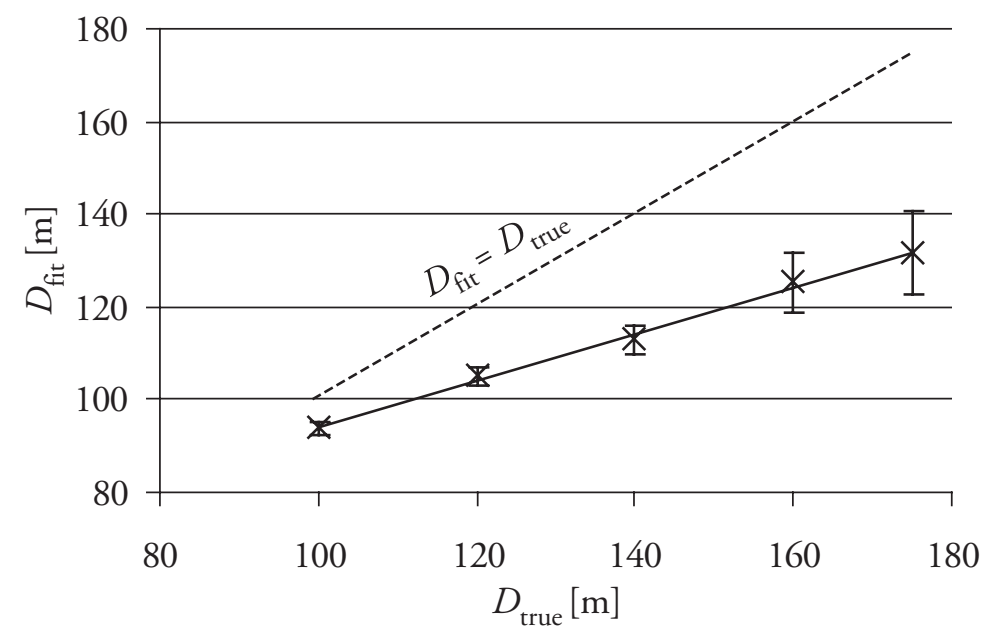

Figure 4. Resulting average fitted source to road distances $\left(D_{\mathrm{fit}}\right)$ as function of actual source to road distance $\left(D_{\text {true }}\right)$, for the unshielded geometry. The dotted line shows the ideal relationship. 
introduced by the model, since the first-order serial expansion (Eq. 5) underestimates the maximum fluence. Using a more sophisticated model would decrease the discrepancy between the fitted and actual distances.

\section{CONCLUSIONS}

The results in this study show that the PSC method is a robust method. The $C D$ is not greatly affected by the semi-shielded geometry, a decrease of about $5 \%$ was found. The $C F$ method is unsuitable to employ as a single search method, at least for the conditions in this study. The $C D$ for the unshielded source is $15 \%$ less than for the PSC method. In addition, shielded sources can be highly detrimental for the $C F$ methods ability to detect the source.

Using the curve fitting technique on the data where the PSC method gives alarm might be a helpful complementary tool. For distances less than about $120 \mathrm{~m}$, the correlation coefficient can be used for deciding if the source is unshielded or not. For unshielded sources, the regression model can be applied to estimate the distance from the road to the source. Increased accuracy of the fitted parameters would be achievable by applying a more sophisticated regression model than the first-order approximation used here.

\section{ACKNOWLEDGEMENT}

This work was supported by The Swedish Radiation Protection Institute, SSI, (project number 710/2846/98).

\section{REFERENCES}

Bristow, Q., 1978. The Application of Airborne Gamma-Ray Spectrometry in the Search for Radioactive Debris from the Russian Satellite Cosmos 954 (Operation "Morning Light"). Current Research, Geological Survey of Canada, Paper 78-1B, 151-162.

Coll, P., 1985. Aspects relating to the calculation of doses in moving radiation field. Health Phys. 49(6), 1139-1146.

Deal, L.J., Doyle, J.F., Burson, Z.G., Boyns, P.K., 1972. Locating the Lost Athena Missile in Mexico by the Aerial Radiological Measuring System (ARMS). Health Phys. 23, 95-98.

European Council (EC), 2003. Council Directive 2003/122/EURATOM of 22 December 2003 on the control of high-activity sealed radioactive sources and orphan sources.

Hjerpe, T., Finck, R.R., Samuelsson, C., 2001. Statistical Data Evaluation in Mobile Gamma Spectrometry: An Optimization of On-Line Search Strategies in the Scenario of Lost Point Sources. Health Phys. 80(6), 563-570.

Hjerpe, T., Samuelsson, C., 2003. A comparison between gross and net count methods when searching for orphan radioactive sources. Health Phys. 84(2), 203-211.

International Atomic Energy Agency (IAEA), 1991. Airborne Gamma Ray Spectrometer Surveying. Vienna. IAEA Technical Reports Series 323. 
International Atomic Energy Agency (IAEA), 2003. Categorization of radioactive sources Revision of IAEA TECDOC 1191, Categorization of radioactive sources. IAEA TECDOC 1344. Vienna.

International Commission on Radiation Units and Measurements (ICRU), 1994. GammaRay Spectrometry in the Environment. Maryland; ICRU Report 53.

Moreira, M.C.F., 1991. Radiological Survey of Goiânia by a Mobile Monitoring Unit. Health Phys 60(1), 81-85. 